\title{
O protagonismo do enfermeiro na equipe de atenção domiciliar
}

\author{
The nursing protagonism in the home care team
}

El protagonismo de enfermería en el equipo de atención a domicilio

Pedro Márlon Martter Moura ${ }^{1 *}$, Adrize Rutz Porto ${ }^{1}$, Cryshna Leticia Kirchesch ${ }^{1}$, Aline Kohler Geppert ${ }^{1}$, Luciana Rodrigues dos Santos ${ }^{1}$, Diana Cecagno ${ }^{1}$, Amanda do Rosário Tavares ${ }^{1}$, Karen Barcelos Lopes ${ }^{1}$, Juliane Portella Ribeiro ${ }^{1}$.

\section{RESUMO}

Objetivo: O presente estudo objetivou conhecer o papel do enfermeiro em equipes multiprofissionais do Programa Melhor em Casa. Métodos: Trata-se de um estudo exploratório, descritivo, de abordagem qualitativa, realizado num Serviço de Atenção Domiciliar vinculado a um Hospital Escola de um município do sul do Brasil. Os participantes do estudo foi de 27, sendo seis médicos, seis enfermeiros, seis técnicos de enfermagem, três auxiliares de enfermagem, dois assistentes sociais, um nutricionista, um psicólogo, um terapeuta ocupacional e um fisioterapeuta. A coleta de dados ocorreu no período de junho a agosto de 2018, por meio de entrevista semiestruturada. Os dados foram analisados pela proposta operativa de análise de conteúdo temática. Resultados: Os resultados demonstraram o protagonismo do enfermeiro na equipe de atenção domiciliar ao assumir a coordenação da equipe e ser referência no serviço, entretanto, tal liderança não é acordada com esse enfermeiro, de modo que tais profissionais referiram se sentirem sobrecarregados com mais essa atividade. Conclusão: Ainda são escassas publicações sobre esse tema, dado a modalidade de atenção domiciliar ser recente no Sistema Único de Saúde, havendo a necessidade de outras investigações para melhor compreender o processo de trabalho das equipes de do Programa Melhor em Casa.

Palavras-chave: Serviços de assistência domiciliar, Enfermagem, Ambiente de trabalho, Equipe de assistência ao paciente.

\begin{abstract}
Objective: This study aimed to understand the role of nurses in multiprofessional teams of the Best at Home Program. Methods: This is an exploratory, descriptive study, with a qualitative approach, carried out in a Home Care Service linked to a Teaching Hospital in a municipality in southern Brazil. The study participants were 27, being six doctors, six nurses, six nursing technicians, three nursing assistants, two social workers, a nutritionist, a psychologist, an occupational therapist and a physical therapist. Data collection took place from June to August 2018, through semi-structured interviews. The data were analyzed using the thematic content analysis operative proposal. Results: The results demonstrated the role of the nurse in the home care team when assuming the coordination of the team and being a reference in the service, however, such leadership is not agreed with this nurse, so that these professionals reported feeling overwhelmed with this activity. Conclusion: There are still few publications on this topic, given that the modality of home care is recent in the Unified Health System, and there is a need for further investigations to better understand the work process of the teams in the Best at Home Program.
\end{abstract}

Key words: Home care services, Nursing, Working environment, Patient care team.

${ }^{1}$ Universidade Federal de Pelotas, Pelotas - RS. *E-mail: pedro.moura@sou.ucpel.edu.br 


\section{RESUMEN}

Objetivo: Este estudio tuvo como objetivo comprender el papel de las enfermeras en los equipos multiprofesionales del Programa Best at Home. Métodos: Se trata de un estudio exploratorio, descriptivo, con abordaje cualitativo, realizado en un Servicio de Atención Domiciliaria vinculado a un Hospital Docente de un municipio del sur de Brasil. Los participantes del estudio fueron 27, siendo seis médicos, seis enfermeros, seis técnicos de enfermería, tres auxiliares de enfermería, dos trabajadores sociales, un nutricionista, un psicólogo, un terapeuta ocupacional y un fisioterapeuta. La recolección de datos se llevó a cabo de junio a agosto de 2018, mediante entrevistas semiestructuradas. Los datos fueron analizados utilizando la propuesta operativa de análisis de contenido temático. Resultados: Los resultados demostraron el rol de la enfermera en el equipo de atención domiciliaria al asumir la coordinación del equipo y ser un referente en el servicio, sin embargo, dicho liderazgo no está acordado con esta enfermera, por lo que estos profesionales informaron sentirse abrumados con esta actividad. Conclusión: aún existen pocas publicaciones sobre este tema, dado que la modalidad de atención domiciliaria es reciente en el Sistema Único de Salud, y se necesitan más investigaciones para comprender mejor el proceso de trabajo de los equipos en el Programa Best at Home.

Palabras clave: Servicios de atención de salud a domicilio, Enfermería, Ambiente de trabajo, Grupo de atención al paciente.

\section{INTRODUÇÃO}

No contexto de saúde brasileira emerge a necessidade de um redirecionamento da atenção em saúde, nos distintos serviços ofertados, tanto no âmbito das instituições privadas, quanto públicas (WEYKAMPJM, et al., 2017). Entre esses serviços, o de Atenção Domiciliar (AD) pode ser uma importante estratégia capaz de auxiliar um reordenamento da atenção na rede de saúde (BRASIL, 2016).

Neste sentido, a instituição da Política Nacional de Atenção Domiciliar (PNAD), incluso o serviço de AD, pela Portaria no 2.029/11, revogada pela de ํㅡ 2.527 de 27 de outubro de 2011, reformulou as normas de habilitação, incentivo financeiro e funcionamento. Após sucessivas revogações, a AD é regulamentada pela Portaria no 825, de 2016 (BRASIL, 2016). A AD, por meio do Programa Melhor em Casa, propõe, entre outros, a desfragmentação da assistência à saúde, indo ao encontro com os princípios propostos pelo Sistema Único de Saúde (SUS) (BRASIL, 2016).

$\mathrm{Na} A D$, como uma modalidade de atenção à saúde, é necessário reconhecer a família como um espaço social. Assim, a Visita Domiciliar (VD) e a Internação Domiciliar (ID), cada uma com seu objetivo e nível de complexidade, são exemplos de ações de cuidado extrahospitalar, possíveis de serem desenvolvidas com o usuário e família (WEYKAMP JM, et al., 2018).

Uma pesquisa indicou que a AD pode evitar inúmeras barreiras de acesso aos serviços, principalmente os de diagnóstico, e ainda, o Programa Melhor em Casa foi mencionado como uma possibilidade de aberturas de portas para outros pontos da rede de atenção à saúde, tornando a atenção ao usuário, individualizada (MORAES WS, et al., 2017).

Estudos enfatizam as vantagens da $A D$, dentre as quais está a possibilidade de uma assistência de qualidade em um ambiente conhecido pelo usuário do serviço, diminuição das internações hospitalares, bem como seus custos, redução de infecções hospitalares (ANDRADE GB, et al., 2019; WEYKAMP JM, et al., 2018, WEYKAMP JM, et al, 2017; SILVA WM, et al., 2017; SILVA LA, et al., 2019).

Outra contribuição da $A D$ é a implementação da assistência pautada no trabalho de equipes interprofissionais, que propõe a participação ativa de equipe, usuários, famílias e comunidade. Nesta ótica, a $A D$ vem sendo consolidada com o comprometimento daqueles profissionais de saúde que, além de integrarem uma equipe multiprofissional, também confiam na eficácia da interação e atuação integrada. Dentre esses profissionais, o enfermeiro é apontado como coordenador, isto é, um organizador e responsável pela eficiência das atividades a serem desenvolvidas, seja em reuniões de equipe, sugerindo estratégias de impacto ao contexto social da população assistida (SILVA LA, et al., 2019). 
Num estudo de revisão de literatura, a atuação do enfermeiro na $A D$ foi identificada como complexa e ampla, sendo permeada por múltiplas atividades, em que as ações relacionais e educacionais se destacam. Além das atividades assistenciais, também foram observadas ações administrativas de supervisão clínica, planejamento e organização das VDs, coordenação do cuidado, gestão de caso (ANDRADE AM, et al., 2017).

Assim, compreendendo a AD como uma modalidade de atenção humanizada e inovadora, com potencial para redirecionar a lógica da assistência fragmentada para a lógica da integralização e, ainda, entendendo que a atuação dos profissionais de saúde assume importante função neste contexto, tem-se como objetivo desta pesquisa conhecer o papel do enfermeiro em equipes multiprofissionais do Programa Melhor em Casa.

\section{MÉTODOS}

Trata-se de um estudo exploratório, descritivo, de abordagem qualitativa, realizado num Serviço de Atenção Domiciliar (SAD) vinculado a um Hospital Escola de um município do sul do Brasil. O Serviço possui três Equipes Multiprofissionais de Atenção Domiciliar (EMAD), sendo uma a cada turno, formando seis EMADs e uma Equipe Multiprofissional de Apoio (EMAP), totalizando 29 profissionais. Cada equipe é responsável pelo atendimento, em média, de 30 pacientes por mês.

O total de participantes desse estudo foi de 27 , uma vez que dois não atenderam aos critérios de inclusão por estarem afastados no período da coleta de dados. Das EMADs, participaram seis médicos, seis enfermeiros, seis técnicos de enfermagem, três auxiliares de enfermagem e dois assistentes sociais e da EMAP, ou equipe de apoio matricial, um nutricionista, um psicólogo, um terapeuta ocupacional e um fisioterapeuta.

A coleta de dados ocorreu no período de junho a agosto de 2018, por meio de entrevista semiestruturada, realizada com cada participante, com duração em média de 20 minutos, tendo sido gravadas e posteriormente transcritas. Os profissionais foram instigados a falarem sobre o trabalho do enfermeiro na equipe multiprofissional.

Os dados foram analisados pela proposta operativa, com a pré-análise, que consistiu na leitura flutuante para gerar impressões iniciais sobre os resultados; exploração do material: recorte, classificação e codificação das informações do material coletado e tratamento e interpretação dos resultados: por meio de abordagem crítica, reflexiva e consistente do material, com inferências acerca dos objetivos da pesquisa (MINAYO MCS, 2014).

O anonimato dos participantes foi garantido por meio de codinomes e numeração de acordo com a sequência da realização das entrevistas. Para os profissionais das EMADs, utilizou-se, por exemplo: Médico (M1, M2, etc.), Enfermeiro (E1, E2, etc.), Técnico ou Auxiliar de Enfermagem (T1, T2, etc.), Assistente Social (AS1, AS2, etc.). Para profissionais da EMAP, utilizou-se a (PEMAP1, PEMAP2, etc.). Os preceitos éticos dessa pesquisa foram baseados na Resolução número 466/2012, que trata das pesquisas envolvendo seres humanos. A pesquisa foi aprovada pelo Comitê de Ética e Pesquisa sob o Certificado de Apresentação para Apreciação Ética número 89470618.0.0000.5316.

\section{RESULTADOS}

No presente estudo, é possível evidenciar a dinâmica do papel do profissional enfermeiro na $A D$, quando se leem os depoimentos dos participantes, que percebem a importância da organização das VDs, além da conexão entre esses profissionais e a comunidade por intermédio da assistência prestada pela enfermeira da equipe:

"Eu chego, a primeira coisa é organizar a rota de visita, para ver quais pacientes estão com previsão de visita pela rotina, quais que precisam de visita extra, quais que tem mais urgência, menos urgência [...]". (E1)

Os relatos demonstram maior envolvimento do enfermeiro na organização das VDs, em relação aos demais membros da equipe, tanto no acompanhamento da equipe com as famílias, como no atendimento às 
necessidades apresentadas. Ainda, por estar mais próximo ao contexto familiar do usuário, o enfermeiro tem condições de atentar para fatores que envolvem as distintas dimensões do ser humano.

Há o entendimento de que o cotidiano de trabalho do enfermeiro sobrecarrega esse profissional e, ainda, há dificuldade de reconhecimento dos demais profissionais da equipe quanto aos atendimentos pertinentes de cada classe:

"[...] o gerenciamento do serviço geralmente é mais da enfermeira. Entre as técnicas têm um acordo, por exemplo: - Ah hoje eu faço isso, amanhã tu fazes tal coisa, a gente se ajuda, então não tem uma divisão exata de tarefas. Mas tem a enfermeira claro, ela que organiza a maior parte da rotina". (T1)

"[...] a sobrecarga de atividades do médico, atendendo outros setores além do SAD [...] sobrecarrega e estressa o enfermeiro, porque temos essa necessidade de sair da casa do paciente com aquela sensação de dever cumprido". (T2)

"[...] enfermeiro é o mestre da equipe, mas isso termina gerando sobrecarga. Daqui a pouco tu estás a fazer uma responsabilidade que não seria tua. Sem a visão do enfermeiro, do cuidado como um todo, a gente não ia conseguir atender bem os pacientes, tanto quando eu quero tirar às vezes uma dúvida, tudo eu recorro basicamente as enfermeiras das equipes, não que o médico não saiba, mas 0 enfermeiro está sempre mais apropriado, então eu acho que ele é um elemento chave da equipe". (PEMAP1)

De acordo com o relato que segue se pode compreender a dinâmica do trabalho do enfermeiro da AD e de que maneira essas relações se estabelecem:

"[...] para mim o enfermeiro é a parte essencial e primordial para o andamento e funcionamento do atendimento dos pacientes [...] Ele organiza as visitas, o sentido de rota, a organização do que está faltando, em questão da agilidade, da padronização dos atendimentos, horário, a quantidade de tempo em cada paciente". (T)

"Às vezes não fica muito claro para mim qual é o papel do enfermeiro, qual é o papel do médico, às vezes eu ouço assim os colegas falando que os enfermeiros acabam fazendo alguns papeis que seriam do médico, mas eu também não sei se o médico tem consciência disso, porque os médicos daqui são bem abertos assim para negociar" (PEMAP)

A participação do usuário/família no plano terapêutico é de grande importância para o sucesso das ações em saúde e essa sintonia positiva só é capaz a partir dos vínculos criados entre os mesmos com a equipe que $o$ assiste. Dentre todos os profissionais, o enfermeiro assume naturalmente papel de destaque, visto que é reconhecido como importante agente no processo de cuidados, orientações, reabilitação e autonomia no momento do estresse da doença.

O compartilhamento de experiências propícios de resgatar não somente a saúde, mas por vezes confortar a integralidade do indivíduo cuidado a partir de suportes emocionais que só a cumplicidade e o vínculo são capazes de estabelecer, é visto nos depoimentos que seguem no que se referem a atuação do enfermeiro:

"[...] [enfermeira] acho ótimo em questão de procedimentos, de curativo, de empatia, de comprometimento, de fazer vínculo com as pessoas, de demonstrar afeto. Eu vejo uma enfermeira que cuida das pessoas, que se importa com elas mesmo, para mim isso é muito valioso". (M2)

"O enfermeiro é aquela pessoa que acolhe muito o paciente, que está ali para estender a mão, além da questão profissional, ele dá um suporte emocional muito grande para o paciente". (AS1) 
Mesmo frente a toda demanda que o enfermeiro possui na equipe de $A D$, a centralização da gestão como líder da equipe de enfermagem confere protagonismo necessário na coordenação das estratégias de ações em saúde, cria vínculos, prioriza planos de cuidados, além de se comprometer com a equipe de forma ética na prestação de atitudes de qualidade, capacitando-a e promovendo estratégias de gestão que atenda às necessidades dos usuários.

Entretanto, o enfermeiro neste estudo, apesar de fazer parte de uma equipe de AD com profissionais dos mais distintos segmentos da área da saúde, é reconhecido empiricamente como o coordenador principal da equipe, papel esse que por vezes sobrecarrega as suas atividades assistenciais/organizacionais e promove uma responsabilidade que não lhes pertence.

Nos depoimentos, na sequência, é observado descontentamento com essa sobrecarga e, por vezes, o incômodo de tomar para si as responsabilidades que deveriam ser da própria equipe de trabalho:

"[...] eles [a equipe] me veem muito mais assim [como coordenadora] [...] 'o que tu achas, tá bom se a gente fizer dessa forma?'. Às vezes se eu não estou, eles me ligam para ver como é que é, [...] como se eu fosse a coordenadora da equipe. Tento sempre partilhar por mais que possa ter essa visão [...] eu me incomodo, sabe, eu acho que não é o meu papel. Na equipe acaba a gente tendo uma responsabilidade maior, e aí tudo acaba ficando a meu critério". (E2)

"[...] eu não gostaria desse papel, que não é de enfermeiro e que foi colocado no meu colo, não foi algo que eu pedi, nem que eu gostaria". (E3)

Destarte, os relatos a seguir descortinam a realidade da organização e da liderança centrada na figura do enfermeiro da $A D$, bem como as decisões acerca das rotinas e das condutas a serem tomadas são compartilhadas com os outros integrantes da equipe de saúde, buscando preservar as competências profissionais de cada membro mesmo, quando essas condutas se tornam comuns entre a equipe:

"[...] o enfermeiro é um líder nato [...] é uma questão da formação deles, embora eu ainda acredite que deve ser dividida as responsabilidades com todos os profissionais". (PEMAP2)

"Então em todas as equipes que eu trabalho, eu vejo o enfermeiro como líder nato dessas equipes, realmente chamando a responsabilidade para si, organizando todas as condutas, sempre respeitando o papel de cada profissional, mas ele assim sabendo de tudo o que acontece com o paciente". (PEMAP3)

"Ela [a enfermeira] é a líder da equipe, mas se ela tem uma dúvida, ela compartilha [...]. Por exemplo, hoje a gente chegou numa casa, ela achou que o paciente estava bem desidratado, conversou com o doutor e pediu a opinião dele e perguntou se podia fazer um soro: foi ela quem sugeriu e ele concordou. Ela toma iniciativa de condutas e ela compartilha [...] e ele [médico] aceita ou não, dependendo de cada situação". (T3).

A seguir, o equilíbrio entre uma liderança compartilhada do enfermeiro e responsabilização de cada membro da equipe pela organização do trabalho é refletida pelos participantes do estudo:

"Por exemplo, para passar uma sonda dentro de um hospital, é uma prescrição que vem do médico e a gente vai lá e passa a sonda. Geralmente aqui, a gente toma uma decisão em conjunto realmente. Eu faço a sugestão, o médico expõe a opinião dele em relação à sondagem e a gente sempre tenta chamar nossa nutricionista para também trazer os motivos que ela acha que deve passar ou não aquela sonda". (E4)

"A questão assim de abertura para se trabalhar interdisciplinar, [...] não que eu vá fazer a função do fisioterapeuta, mas eu posso entrar no campo dele e fazer o comum, $e$ isso na atenção domiciliar é o que eu mais gosto, porque eu vejo que dentro do hospital a gente não consegue fazer muito isso". (E5) 


\section{DISCUSSÃO}

$A A D$ é um modelo de cuidado que visa a reorganização do processo de trabalho em saúde, tendo como um de seus objetivos a redução da demanda hospitalar, bem como o período de internação, além promover conforto, segurança ao paciente, bem-estar e redução de gastos (RAJÃO FL e MARTINS M, 2020; LOPES GVDO, et al., 2017). Este tipo de assistência foi considerado uma modalidade integral de cuidado à saúde, com maior probabilidade de uma atenção contínua (VALARISTINO JM, et al., 2019).

Além dessa modalidade ofertar ao paciente a possibilidade e humanização da assistência, aumenta o vínculo da equipe e a participação familiar no processo de reabilitação do usuário e por ser um serviço que aumenta a desospitalização, favorece assim a maior disponibilidade de leitos hospitalares para casos mais graves, reduzindo significativamente o número de infecção hospitalar (SANTOS DLFS, 2019).

Dentre os profissionais da equipe de saúde, o enfermeiro é identificado como aquele que, inúmeras vezes, organiza e se responsabiliza, pelo processo de trabalho da equipe, propondo e executando estratégias que objetivam impacto ao contexto social da população assistida e consequentemente à realidade de vida e saúde desses usuários (SILVA LA, et al., 2019). Além disso, uma vez que a aproximação que a equipe de enfermagem possui junto ao usuário, sua família e comunidade e o vínculo de cuidados que surgem durante as VDs ultrapassam a linha profissional, de modo que tal fato pode ampliar a conquista da autonomia dessas famílias com a sua saúde. (CONCEIÇÃO AS, et al., 2019)

A Resolução COFEn no 0464/2014, que visa a normatização da atuação da equipe de enfermagem na atenção domiciliar, estabelece que é competência privativa do enfermeiro dimensionar a equipe de enfermagem; planejar, organizar, supervisionar e avaliação a prestação da assistência de enfermagem; organizar e coordenar as condições ambientais, equipamentos e materiais necessários à produção do cuidado competente, resolutivo e seguro; atuar de forma contínua na capacitação da equipe de enfermagem que atua na realização de cuidados nesse ambiente; executar os cuidados de enfermagem de maior complexidade técnico-científica e que demandem a necessidade de tomar decisões imediatas (BRASIL, 2014).

Os profissionais de enfermagem têm sido apontados como um profissional de referência no cuidado domiciliar, pois passam segurança e confiança aos cuidadores através de seus conhecimentos, uma vez que são responsáveis pela coordenação do plano de cuidados e construção de vínculos (PROCÓPIO LCR, et al., 2019).

A VD é uma estratégia de criação de vínculo, uma vez que acompanha os usuários do serviço em seu lar, além de permitir que o profissional de saúde conheça a realidade, na qual está inserido o usuário e sua família (ROCHA KB, et al., 2017). A VD requer disponibilidade, organização e comprometimento dos profissionais que a realizam, visto que é uma atividade que, além da assistência qualificada, humanizada e diferenciada frente às necessidades específicas, oportuniza ao usuário dispositivos promotores de saúde (SILVA LA, et al., 2019).

Neste sentido, a VD, quando bem planejada, e discutida entre os membros da equipe, pode ampliar as chances de atender as necessidades individuais de cada usuário e sua família (SILVA WM, et al., 2017). Nesse contexto, o enfermeiro realiza ações que vão além das suas responsabilidades, sobrecarregando a sua jornada de trabalho, seja pela falta de profissionais, de materiais ou pela necessidade de um diagnóstico de enfermagem efetivo, assim como pelas constantes capacitações dos cuidadores. Esses percalços propiciam por vezes a inversão entre os papeis dos diferentes trabalhadores da equipe o que, rotineiramente, aumenta a demanda de atendimentos do enfermeiro (SILVA WM, et al., 2017).

Em consonância com os outros profissionais da equipe de saúde, os enfermeiros possuem grande capacidade de tomada de decisão com embasamento técnico científico que, a partir da proximidade e da empatia criada com os usuários da $A D$, confere autonomia e responsabilidade diferenciada do restante da equipe contemplando um dos grandes pilares para o êxito das atividades em saúde (ANDRADE AM, et al., 2017).

O enfermeiro é visto como peça imprescindível na coordenação e na gestão do trabalho da equipe de enfermagem e na capacitação dos cuidadores, da família e da comunidade devido à perspicácia de adaptar- 
se aos mais diferentes contextos sociais, vencendo desafios e incorporando a cientificidade adquirida durante a sua vida profissional com a realidade de cada família (ANDRADE AM, et al., 2019).

Uma das rotinas do enfermeiro na VD é ter organização e objetivos claros sobre o que procura na intervenção na vida das famílias que atuam. A disponibilidade de tempo, a qualidade do atendimento e as dificuldades que serão enfrentadas não devem dificultar as estratégias elaboradas com a equipe de saúde, preservando o atendimento de qualidade e excelência por consulta de enfermagem minuciosa, avaliação do contexto social e das adversidades a serem enfrentadas (GOMES MFP, et al., 2015).

Dentre todos os profissionais, o enfermeiro naturalmente assume papel de destaque visto que é reconhecido como principal agente no processo de cuidados, orientações, reabilitação e autonomia no momento da doença (SILVA LA, et al., 2017).

Os vínculos estabelecidos fazem parte do contexto domiciliar e promovem sensação de confiança e consequentemente de autonomia dos usuários com a equipe e gradativamente maior comprometimento com a saúde como um todo, despertando a consciência da prevenção e do autocuidado numa visão ampliada entre saúde e prevenção (SILVA LA, et al., 2017)

Em consonância, os profissionais enfermeiros possuem grande capacidade de tomada de decisão com embasamento técnico-científico, e a partir da proximidade e da empatia que cria com os usuários da atenção domiciliar, conferindo autonomia e responsabilidade diferenciada do restante da equipe e contempla um dos grandes pilares para o êxito das atividades em saúde (ANDRADE AM, et al., 2017).

O profissional de enfermagem possui um papel fundamental dentro da $A D$, assegurando as condutas adequadas para tratamento e reabilitação da saúde, uma vez que existe uma construção histórica do papel da enfermagem através do cuidado de forma inerente em sua atuação, pautada no amor, caridade e altruísmo aos indivíduos (RIBEIRO DFS, et al., 2016).

A enfermagem é a classe profissional que dedica mais tempo dentro da $A D$ e realiza o maior número de procedimentos. Dentro desse cenário, a atuação do enfermeiro engloba inúmeras possibilidades de atuação, sendo elas: consultas primárias, cuidado com curativos, estomias, sondagem, cateteres, drenos e administração e orientação quanto aos medicamentos prescritos (RIBEIRO DFS, et al., 2017).

A interdisciplinaridade dentro da $A D$ é extremamente relevante para a recuperação do estado de saúde dos pacientes, uma vez que a assistência ocorre de maneira integral através do conhecimento individual de cada profissional. Essa articulação das diferentes práticas e saberes permite ir além das práticas biologicistas (RIBEIRO DFS e PÉRRISSÉ G, 2017).

Reconhecer os diversos papeis e a responsabilidade de cada membro da equipe, além de evitar ruídos no ambiente de trabalho, também delimita de forma sincrônica, a importância de cada profissional no cuidado e nas ações de saúde do usuário e de sua família. A equipe multiprofissional busca trabalhar com os mais diferentes saberes, complementam o cuidado e fortalecem a AD com os mais variados diagnósticos e quadros clínicos (COSTA IK, et al., 2019).

\section{CONCLUSÃO}

A atuação técnica do enfermeiro não é claramente diferenciada pelos demais profissionais da saúde, quando se considera os procedimentos que o técnico de enfermagem realiza. Apesar disso, a equipe de saúde reconhece o protagonismo do enfermeiro e a liderança nos cuidados e constituição de vínculos aos usuários do serviço, atribuindo-lhe o papel de coordenador da equipe. Tal incumbência, é vista, principalmente, pelos enfermeiros, como uma responsabilidade que não foi voluntariamente escolhida, o que Ihe gera acúmulo de tarefas e, por consequência, sobrecarga de trabalho. Embora a presente pesquisa aborde o serviço de AD de um município, notou-se que converge com outras investigações, ainda que sejam escassas sobre esse tema, dado a modalidade de AD ser recente no SUS. Assim, outras investigações se fazem necessárias para melhor compreender o processo de trabalho das equipes de AD do Programa Melhor em Casa. 


\section{REFERÊNCIAS}

1. ANDRADE AM, et al. Nursing practice in home care: an integrative literature review. Revista Brasileira de Enfermagem, 2017; 70(1):199-208.

2. ANDRADE AM, et al. Prática da enfermeira na atenção domiciliar: o cuidado mediado pela reflexividade. Revista Brasileira de Enfermagem, 2019; 72(4): 956-63.

3. ANDRADE GB, et al. Palliative care and the importance of communication between nurse and patient, family and caregiver. Revista de Pesquisa: Cuidado é Fundamental, 2019; 11(1): 713-17.

4. BRASIL. Ministério da Saúde. Portaria no 825, de 25 de abril de 2016. Redefine a Atenção Domiciliar no âmbito do Sistema Único de Saúde (SUS) e atualiza as equipes habilitadas. 2016. Disponível em: https://portalarquivos.saude.gov.br/images/pdf/2016/abril/27/PORTARIA-825.pdf Acessado em: 07 de maio de 2021.

5. CONCEIÇÃO A, et al. Ações da enfermeira na visita domiciliar da atenção básica. Revista Eletrônica Acervo Saúde, $2014 ; 20$, e441-41.

6. COSTA IK, et al. O papel de cada profissional de saúde na atenção domiciliar: uma revisão da literatura. Relatos de casos, 2019; 63(4): 455-61.

7. GOMES MFP, et al. Atenção domiciliar do enfermeiro na estratégia de saúde da família. Mundo Saúde, 2015; 39(4): 470-5.

8. LOPES GVDO, et al. Atenção Domiciliar na Estratégia Saúde da Família: avaliação do grau de implantação em Camaçari (BA). Saúde em Debate, 2017; 41(3): 241-54

9. MINAYO MCS. O desafio do conhecimento: pesquisa qualitativa em saúde. 30ª ed. São Paulo: Hucitec; 2016.

10. COFEN. Conselho Federal de Enfermagem. Resolução no 0464/2014. Normatiza a atuação da equipe de enfermagem na atenção domiciliar. 2014. Disponível em: http://www.cofen.gov.br/resolucao-cofen-no04642014_27457.html Acessado em: 07 de maio de 2021.

11. MORAES WS, et al. Assistência de enfermagem em atenção domiciliar no nível primário de atenção à saúde. Caderno de Graduação-Ciências Biológicas e da Saúde-UNIT-ALAGOAS, 2017; 4(1): 11.

12. PROCÓPIO LCR, et al. A Atenção Domiciliar no âmbito do Sistema Único de Saúde: desafios e potencialidades. Saúde em Debate, 2019; 43(121): 592-604.

13. RAJÃO FL, MARTINS M. Atenção Domiciliar no Brasil: estudo exploratório sobre a consolidação e uso de serviços no Sistema Único de Saúde. Ciência \& Saúde Coletiva, 2020; 25(5): 1863-77.

14. RIBEIRO DFS, et al. Atribuições do enfermeiro em um programa de atenção domiciliar do Sistema Único de Saúde. Revista de Atenção à Saúde, 2017; 15(52): 56-60.

15. RIBEIRO DFS, PÉRRISSÉ G. O cuidado do enfermeiro na atenção domiciliar: o caso do programa de atenção domiciliar ao idoso do município do rio de janeiro. Revista Rede de Cuidados em Saúde, 2016; 10(3): 1-7.

16. ROCHA KB, et al. A visita domiciliar no contexto da saúde: uma revisão de literatura. Psic., Saúde \& Doenças, 2017; 18(1): 170-85.

17. SANTOS DFLS. Assistência domiciliar: Reflexão acerca da sua abordagem na literatura. Trabalho de Conclusão de Curso (Bacharelado em Enfermagem) - Faculdade Nova Esperança de Mossoró. Escola de Enfermagem Nova Esperança, Mossoró, Rio Grande do Norte, 2019; 33 p.

18. SILVA LA, et al. Atenção domiciliar: revisão integrativa das finalidades do trabalho da enfermagem. Revista Científica da Saúde, 2019; 1(1): 31-40.

19. VALARISTINO JM, et al. Atuação do enfermeiro na atenção domiciliar: revisão narrativa. Artigos@, $2019 ; 12: e 2567$.

20. WEYKAMP JM, et al. Cuidados do enfermeiro ao usuário nas modalidades de atenção domiciliar. Revista de Pesquisa: Cuidado é Fundamental, 2018; 10(1): 1130-40.

21. WEYKAMP JM, et al. Quality of life and chronic renal failure. Revista de Pesquisa: Cuidado é Fundamental, 2017; 9(1): 1113-20. 